\title{
Technological Change and Risk Adjustment: Benefit Design Incentives in Medicare Part D Online Appendix
}

\author{
Colleen Carey \\ Colleen.Carey@cornell.edu
}

\section{Appendix}

\section{A Subsidization Scheme for Medicare Part D Plans}

A Part D plan $i$ who enrolls beneficiary $j$ will receive four types of payments for that beneficiary:

$$
\text { Payments }_{i j}=\underbrace{\left(D P_{i j}+G P_{i j}-\text { prem }_{i}\right)}_{\text {Direct Subsidy }}+\text { prem }_{i}+R I_{i j}
$$

where $D P_{j}$ is the diagnosis-specific risk adjustment for beneficiary $j$ in plan $i$

$G P_{i j}$ is the demographic-specific risk adjustment for beneficiary $j$ in plan $i$

prem $_{i}$ is chosen plan $i$ 's premium paid by each beneficiary

$R I_{i j}$ is the government reinsurance payment for beneficiary $j$ in chosen plan $i$

The Direct Subsidy contains the risk adjustment that the plan receives for beneficiary $j$. The diagnosisspecific portion is the sum of risk adjustment over the individual's diagnoses, scaled up or down by the plan's bid.

$$
D P_{i j}=\frac{b i d_{i}}{N A B} \sum_{x} W_{x} D_{j x}
$$

$W_{x}$ are the risk adjustment amounts (in dollars) described in Section II. 1 and $D_{j x}$ is 1 if beneficiary $j$ has diagnosis $x$. These weights are inflated by the ratio of the plan's bid to the national average bid for the year. Demographic-specific risk adjustment $G P_{i j}$ are computed in the same way for the demographic categories described in Section $\amalg .1$

Insurance plans also receive premiums and reinsurance payments. Plan $i$ 's premium prem $i$ is subtracted from the Direct Subsidy and then collected directly from beneficiaries. Reinsurance payments reimburse plans directly for $80 \%$ of plan liabilities in the catastrophic zone. The government makes two other payments to plans that I ignore in my empirical strategy. Firstly, because low-income beneficiaries pay reduced copays, the Low-Income Cost-Sharing Subsidy reimburses plans directly for the difference between the reduced copay and the plan's stated copay. I can ignore this payment because plan expenditure is the same regardless of a beneficiary's low-income status. Secondly, a Risk Corridor payment partially offsets the losses of any plan whose total expenditure exceeds its total receipts by five percent. If instead an plan's total receipts exceed its expenditure by $5 \%$, the insurer remits part of its profits to the government under the rules of the Risk Corridor. Since risk corridor payments apply at the plan level rather than the beneficiary level, I cannot incorporate them into my adjustments.

\section{B Adjustments to Raw Plan Expenditure}

In the Medicare prescription drug claims, I observe the plan's raw expenditure on each beneficiary. I make four modifications to this expenditure in order to isolate the portion of expenditure that corresponds to diagnosis-specific risk adjustment.

\footnotetext{
${ }^{1}$ To reiterate, $W_{x}$ in this paper are the weights in Robst et al. (2007) times the 2009 national average bid and divided by the 2009 upcoding normalization factor.
} 
1. I subtract reinsurance payments. Reinsurance payments are simply calculated as $80 \%$ of the plan's expenditure above the catastrophic threshold.

2. I subtract demographic risk adjustment.

3. I subtract supplemental premiums collected by plans offering enhanced benefits. The supplemental premium is paid by beneficiaries but not subtracted from the Direct Subsidy, therefore it does not drop out of total payments.

4. Finally, I divide by $\frac{b i d_{i}}{N A B}$. This rescales plan expenditure by overall plan generosity to make expenditure incurred by plans of varying generosity comparable.

Modified expenditure $\widetilde{E_{j}}$ is the dependent variable in Equation 2

\section{Use of Datasources Over Time}

The table below describes the timeline of data used to for risk adjustment and this paper's measurement and estimation. The first column shows that the Part D risk adjustment calibration data comes from 2000 (disabled Medicaid beneficiaries used to represent disabled Medicare beneficiaries) and 2002 (Federal retirees used to represent elderly Medicare beneficiaries). I collect the cumulative technological change (entrants and new generics) between 2003 and 2008, inclusive. Medical and prescription drug claims for a 5\% sample of Medicare beneficiaries are used to link drugs to diagnoses (Section V.3). Risk adjustment payments are based on diagnoses from the previous calendar year, so in estimation of Equation 2, I use diagnoses from 2008 and modified expenditure from 2009. We call the resulting coefficient treatment costs for 2009. The difference between risk adjustment in 2009 and treatment costs in 2009 is profitability for 2009, which is the independent variable in the estimation of Equation 4. I use 2009 profitability to explain benefit design outcomes in 2010; finally, this equation is weighted by expenditure on drugs in Medicare Advantage in 2009.

Table A1: Datasources Over Time

\begin{tabular}{|c|c|c|c|c|c|}
\hline & $\begin{array}{l}\text { Risk adjustment } \\
\text { calibration data }\end{array}$ & $\begin{array}{l}\text { Stock of Tech. } \\
\text { Change from FDA }\end{array}$ & $\begin{array}{l}\text { Linking Drugs } \\
\text { to Diagnoses }\end{array}$ & $\begin{array}{l}\text { Measuring } \\
\text { Profitability }\end{array}$ & $\begin{array}{l}\text { Effect of Profitability } \\
\text { on Outcomes }\end{array}$ \\
\hline 2000 & Medicaid & & \multirow{12}{*}{$\begin{array}{l}\text { RHS: diagnoses } \\
\text { LHS: drug choices } \\
\text { RHS: diagnoses } \\
\text { LHS: drug choices } \\
\text { RHS: diagnoses } \\
\text { LHS: drug choices }\end{array}$} & & \multirow{12}{*}{$\begin{array}{l}\text { RHS: profitability } \\
\text { weights: MA spending } \\
\text { LHS: outcomes }\end{array}$} \\
\hline 2001 & & & & & \\
\hline 2002 & Federal Retiree & & & & \\
\hline 2003 & & $\mathrm{X}$ & & & \\
\hline 2004 & & $\mathrm{X}$ & & & \\
\hline 2005 & & $\mathrm{X}$ & & & \\
\hline 2006 & & $\mathrm{X}$ & & & \\
\hline 2007 & & $\mathrm{X}$ & & & \\
\hline $200 r$ & & $\Lambda$ & & & \\
\hline 2008 & & $\mathrm{X}$ & & RHS: diagnoses & \\
\hline 2009 & & & & LHS: spending & \\
\hline 2010 & & & & & \\
\hline
\end{tabular}


Table A2: Effect of Profitability on Benefit Design: Each Technological Change Instrument Singly

\begin{tabular}{|c|c|c|c|c|c|c|}
\hline \multicolumn{7}{|c|}{ IV: Entrants } \\
\hline & $\begin{array}{l}\text { covered } \\
\text { (p.p) }\end{array}$ & $\begin{array}{l}\text { out-of-pocket } \\
\operatorname{cost}(\$)\end{array}$ & $\operatorname{copay}(\$)$ & coins. $(\$)$ & tier & $\begin{array}{l}\text { OOP is } \\
\text { coins? (p.p.) }\end{array}$ \\
\hline Profitability & $\begin{array}{l}-0.0103 \\
(0.0179)\end{array}$ & $\begin{array}{c}0.2031 \\
(0.5599)\end{array}$ & $\begin{array}{c}0.0079 \\
(0.0315)\end{array}$ & $\begin{array}{l}-0.5388 \\
(0.9029)\end{array}$ & $\begin{array}{c}0.0016 \\
(0.0020)\end{array}$ & $\begin{array}{c}0.0584 \\
(0.0583)\end{array}$ \\
\hline $\begin{array}{l}\text { Entrants } \\
\text { F }\end{array}$ & $\begin{array}{c}-34.12 \\
(10.08)^{* * *} \\
11\end{array}$ & $\begin{array}{c}-12.96 \\
(6.64)+ \\
4\end{array}$ & $\begin{array}{c}\text { First Stage } \\
-14.58 \\
(3.71)^{* * *} \\
15\end{array}$ & $\begin{array}{c}-15.61 \\
(11.73) \\
2\end{array}$ & $\begin{array}{c}-12.96 \\
(6.64)+ \\
4\end{array}$ & $\begin{array}{c}-12.96 \\
(6.64)+ \\
4\end{array}$ \\
\hline \multicolumn{7}{|c|}{ IV: New Generics } \\
\hline & $\begin{array}{l}\text { covered } \\
\text { (p.p) }\end{array}$ & $\begin{array}{l}\text { out-of-pocket } \\
\text { cost }(\$)\end{array}$ & $\operatorname{copay}(\$)$ & coins. $(\$)$ & tier & $\begin{array}{l}\text { OOP is } \\
\text { coins? (p.p.) }\end{array}$ \\
\hline Profitability & $\begin{array}{c}0.0102 \\
(0.0330)\end{array}$ & $\begin{array}{c}-0.4471 \\
(0.3302)\end{array}$ & $\begin{array}{c}-0.0808 \\
(0.0173)^{* * *} \\
\text { First Stage }\end{array}$ & $\begin{array}{c}0.1404 \\
(0.6581)\end{array}$ & $\begin{array}{c}-0.0034 \\
(0.0007)^{* * *}\end{array}$ & $\begin{array}{c}-0.077 \\
(0.0179)^{* * *}\end{array}$ \\
\hline $\begin{array}{l}\text { New Generics } \\
\mathrm{F}\end{array}$ & $\begin{array}{c}4.91 \\
(0.81)^{* * *} \\
36\end{array}$ & $\begin{array}{c}9.45 \\
(1.41)^{* * *} \\
45\end{array}$ & $\begin{array}{c}6.74 \\
(0.83)^{* * *} \\
66\end{array}$ & $\begin{array}{c}11.59 \\
(2.47)^{* * *} \\
22\end{array}$ & $\begin{array}{c}9.45 \\
(1.41)^{* * *} \\
45\end{array}$ & $\begin{array}{c}9.45 \\
(1.41)^{* * *} \\
45\end{array}$ \\
\hline \multicolumn{7}{|c|}{ IV: Expenditure on Entrants } \\
\hline & $\begin{array}{l}\text { covered } \\
\text { (p.p) }\end{array}$ & $\begin{array}{l}\text { out-of-pocket } \\
\text { cost }(\$)\end{array}$ & $\operatorname{copay}(\$)$ & coins. $(\$)$ & tier & $\begin{array}{l}\text { OOP is } \\
\text { coins? (p.p.) }\end{array}$ \\
\hline Profitability & $\begin{array}{c}0.2179 \\
(0.0768)^{* *}\end{array}$ & $\begin{array}{c}-3.7528 \\
(19.1437)\end{array}$ & $\begin{array}{c}-0.1765 \\
(0.1445) \\
\text { First Stage }\end{array}$ & $\begin{array}{c}10.1602 \\
(34.9727)\end{array}$ & $\begin{array}{c}0.0116 \\
(0.1023)\end{array}$ & $\begin{array}{l}-0.6785 \\
(3.5075)\end{array}$ \\
\hline$\$$ on Entrants & $\begin{array}{c}0.16 \\
(0.17)\end{array}$ & $\begin{array}{c}0.01 \\
(0.10)\end{array}$ & $\begin{array}{l}-0.09 \\
(0.06)\end{array}$ & $\begin{array}{c}0.04 \\
(0.18)\end{array}$ & $\begin{array}{c}0.01 \\
(0.10)\end{array}$ & $\begin{array}{c}0.01 \\
(0.10)\end{array}$ \\
\hline $\mathrm{F}$ & 1 & 0 & 2 & 0 & 0 & 0 \\
\hline \multicolumn{7}{|c|}{ IV: Takers of New Generics } \\
\hline & $\begin{array}{l}\text { covered } \\
\text { (p.p) }\end{array}$ & $\begin{array}{l}\text { out-of-pocket } \\
\text { cost }(\$)\end{array}$ & $\operatorname{copay}(\$)$ & coins. $(\$)$ & tier & $\begin{array}{l}\text { OOP is } \\
\text { coins? (p.p.) }\end{array}$ \\
\hline Profitability & $\begin{array}{c}0.0371 \\
(0.0216)+\end{array}$ & $\begin{array}{c}-0.5379 \\
(0.2854)+\end{array}$ & $\begin{array}{c}-0.0624 \\
(0.0123)^{* * *} \\
\text { First Stage }\end{array}$ & $\begin{array}{l}-0.2528 \\
(0.5790)\end{array}$ & $\begin{array}{c}-0.0028 \\
(0.0005)^{* * *}\end{array}$ & $\begin{array}{c}-0.0679 \\
(0.0143)^{* * *}\end{array}$ \\
\hline Takers of & 13.71 & 20.8 & 15.43 & 24.83 & 20.8 & 20.8 \\
\hline New Generics & $(3.31)^{* * *}$ & $(2.58)^{* * *}$ & $(1.59)^{* * *}$ & $(3.95)^{* * *}$ & $(2.58)^{* * *}$ & $(2.58)^{* * *}$ \\
\hline $\mathrm{F}$ & 17 & 65 & 94 & 40 & 65 & 65 \\
\hline Sample & all & covered & $\begin{array}{l}\text { covered \& } \\
\text { OOP is copay }\end{array}$ & $\begin{array}{l}\text { covered \& } \\
\text { OOP is coins? }\end{array}$ & covered & covered \\
\hline
\end{tabular}




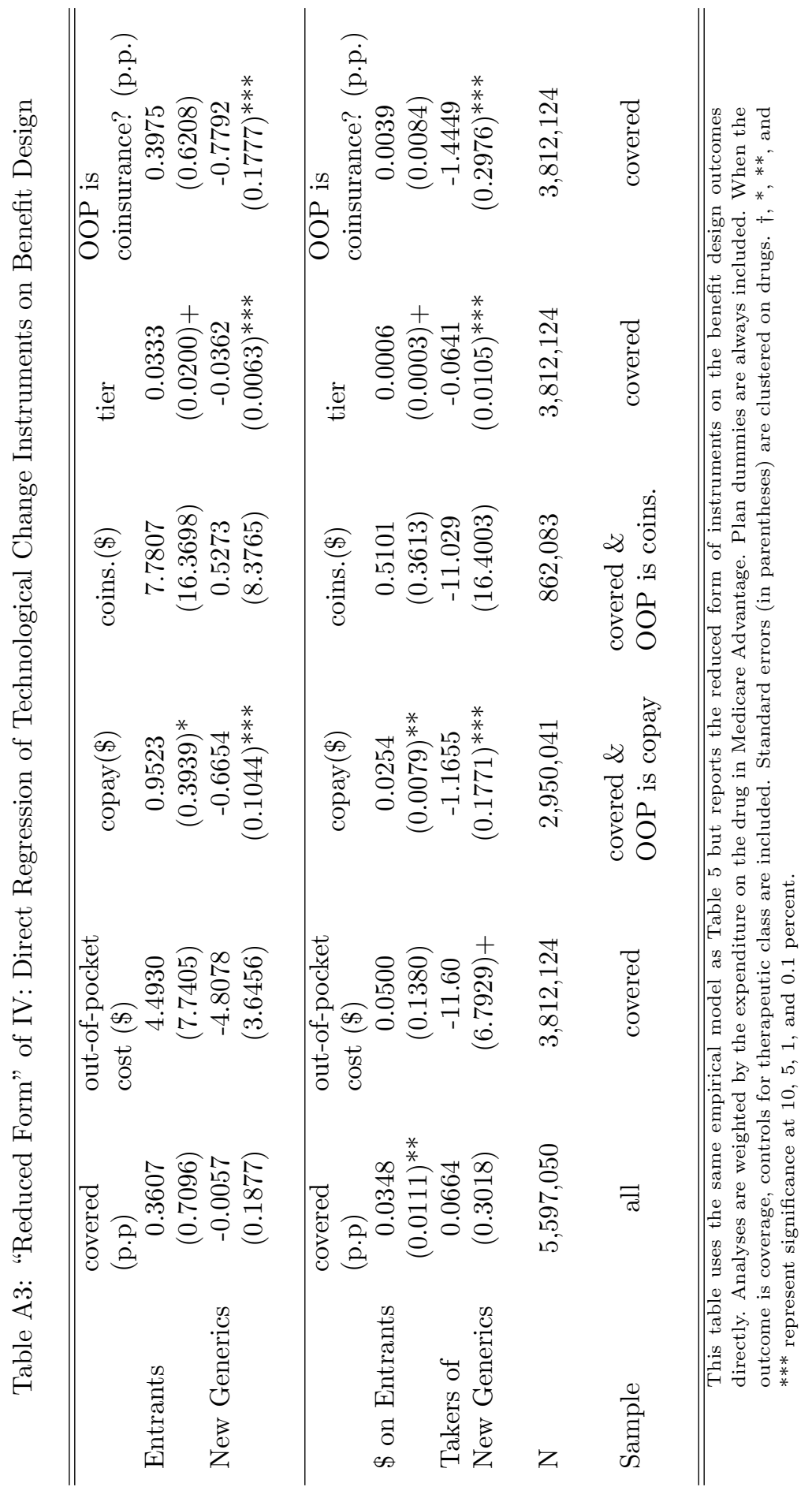


Table A4: First Stage for Table 6

\begin{tabular}{|c|c|c|c|c|c|c|}
\hline \multicolumn{7}{|c|}{ First Stage for Brands } \\
\hline & $\begin{array}{l}\text { covered } \\
\text { (p.p) }\end{array}$ & $\begin{array}{l}\text { out-of-pocket } \\
\text { cost }(\$)\end{array}$ & $\operatorname{copay}(\$)$ & coins. $(\$)$ & tier & $\begin{array}{l}\text { OOP is } \\
\text { coins? (p.p.) }\end{array}$ \\
\hline \multicolumn{7}{|c|}{ IV: Entrants and New Generics } \\
\hline Entrants & $\begin{array}{c}-85.38 \\
(40.59)^{*}\end{array}$ & $\begin{array}{c}-4.48 \\
(15.42)\end{array}$ & $\begin{array}{c}-28.58 \\
(8.18)^{* * *}\end{array}$ & $\begin{array}{c}4.93 \\
(17.03)\end{array}$ & $\begin{array}{c}-4.48 \\
(15.42)\end{array}$ & $\begin{array}{l}-4.48 \\
(15.42)\end{array}$ \\
\hline New Generics & $\begin{array}{c}19.2 \\
(6.56)^{* *}\end{array}$ & $\begin{array}{c}10.63 \\
(1.76)^{* * *}\end{array}$ & $\begin{array}{c}8.85 \\
(1.27)^{* * *}\end{array}$ & $\begin{array}{c}14.61 \\
(3.15)^{* * *}\end{array}$ & $\begin{array}{c}10.63 \\
(1.76)^{* * *}\end{array}$ & $\begin{array}{c}10.63 \\
(1.76)^{* * *}\end{array}$ \\
\hline $\mathrm{F}$ & 14 & 21 & 25 & 12 & 21 & 21 \\
\hline \multicolumn{7}{|c|}{ IV: Expenditure on Entrants and Takers of New Generics } \\
\hline$\$$ on Entrants & $\begin{array}{c}1.64 \\
(0.92)+\end{array}$ & $\begin{array}{c}0.23 \\
(0.27)\end{array}$ & $\begin{array}{c}-0.21 \\
(0.13)\end{array}$ & $\begin{array}{c}0.35 \\
(0.29)\end{array}$ & $\begin{array}{c}0.23 \\
(0.27)\end{array}$ & $\begin{array}{c}0.23 \\
(0.27)\end{array}$ \\
\hline Takers of & -12.04 & 18.87 & 13.29 & 26.14 & 18.87 & 18.87 \\
\hline New Generics & $(11.85)$ & $(3.70)^{* * *}$ & $(2.07)^{* * *}$ & $(5.76)^{* * *}$ & $(3.70)^{* * *}$ & $(3.70)^{* * *}$ \\
\hline $\mathrm{F}$ & 22 & 34 & 35 & 16 & 34 & 34 \\
\hline \multicolumn{7}{|c|}{ First Stage for Generics } \\
\hline & $\begin{array}{l}\text { covered } \\
\text { (p.p) }\end{array}$ & $\begin{array}{l}\text { out-of-pocket } \\
\text { cost }(\$)\end{array}$ & $\operatorname{copay}(\$)$ & coins. $(\$)$ & tier & $\begin{array}{l}\text { OOP is } \\
\text { coins? (p.p.) }\end{array}$ \\
\hline \multicolumn{7}{|c|}{ IV: Entrants and New Generics } \\
\hline Entrants & $\begin{array}{c}-89.65 \\
(16.73)^{* * *}\end{array}$ & $\begin{array}{c}-30.02 \\
(8.78)^{* * *}\end{array}$ & $\begin{array}{c}-31.72 \\
(8.48)^{* * *}\end{array}$ & $\begin{array}{c}-23.69 \\
(10.89)^{*}\end{array}$ & $\begin{array}{c}-30.02 \\
(8.78)^{* * *}\end{array}$ & $\begin{array}{c}-30.02 \\
(8.78)^{* * *}\end{array}$ \\
\hline New Generics & 17.18 & 8.76 & 8.96 & $\begin{array}{c}(10.09) \\
7.54\end{array}$ & 8.76 & 8.76 \\
\hline & $(2.62)^{* * *}$ & $(1.17)^{* * *}$ & $(1.17)^{* * *}$ & $(1.44)^{* * *}$ & $(1.17)^{* * *}$ & $(1.17)^{* * *}$ \\
\hline & 27 & 33 & 37 & 17 & 33 & 33 \\
\hline \multicolumn{7}{|c|}{ IV: Expenditure on Entrants and Takers of New Generics } \\
\hline$\$$ on Entrants & $\begin{array}{l}-0.12 \\
(0.38)\end{array}$ & $\begin{array}{c}-0.18 \\
(0.07)^{*}\end{array}$ & $\begin{array}{c}-0.18 \\
(0.07)^{* *}\end{array}$ & $\begin{array}{c}-0.19 \\
(0.09)^{*}\end{array}$ & $\begin{array}{c}-0.18 \\
(0.07)^{*}\end{array}$ & $\begin{array}{l}-0.18 \\
(0.07)^{*}\end{array}$ \\
\hline Takers of & 11.41 & 12.85 & 12.75 & 13.46 & 12.85 & 12.85 \\
\hline New Generics & $(2.79)^{* * *}$ & $(1.92)^{* * *}$ & $(1.72)^{* * *}$ & $(2.43)^{* * *}$ & $(1.92)^{* * *}$ & $(1.92)^{* * *}$ \\
\hline $\mathrm{F}$ & 17 & 22 & 28 & 15 & 22 & 22 \\
\hline \multicolumn{7}{|c|}{ First Stage for Protected } \\
\hline & $\begin{array}{l}\text { covered } \\
\text { (p.p) }\end{array}$ & $\begin{array}{l}\text { out-of-pocket } \\
\text { cost }(\$)\end{array}$ & $\operatorname{copay}(\$)$ & coins. $(\$)$ & tier & $\begin{array}{l}\text { OOP is } \\
\text { coins? (p.p.) }\end{array}$ \\
\hline \multicolumn{7}{|c|}{ IV: Entrants and New Generics } \\
\hline Entrants & $\begin{array}{c}-30.51 \\
(7.55)^{* * *}\end{array}$ & $\begin{array}{c}-51.81 \\
(9.14)^{* * *}\end{array}$ & $\begin{array}{c}-26.32 \\
(5.54)^{* * *}\end{array}$ & $\begin{array}{c}-76.15 \\
(13.34)^{* * *}\end{array}$ & $\begin{array}{c}-51.81 \\
(9.14)^{* * *}\end{array}$ & $\begin{array}{c}-51.81 \\
(9.14)^{* * *}\end{array}$ \\
\hline New Generics & $\begin{array}{c}20.58 \\
(7.12)^{* *}\end{array}$ & $\begin{array}{c}57.51 \\
(12.58)^{* * *}\end{array}$ & $\begin{array}{c}29.56 \\
(7.31)^{* * *}\end{array}$ & $\begin{array}{c}73.29 \\
(17.29)^{* * *}\end{array}$ & $\begin{array}{c}57.51 \\
(12.58)^{* * *}\end{array}$ & $\begin{array}{c}57.51 \\
(12.58)^{* * *}\end{array}$ \\
\hline $\mathrm{F}$ & 18 & 19 & 12 & 23 & 19 & 19 \\
\hline \multicolumn{7}{|c|}{ IV: Expenditure on Entrants and Takers of New Generics } \\
\hline$\$$ on Entrants & $\begin{array}{c}0.24 \\
(0.29)\end{array}$ & $\begin{array}{c}-0.71 \\
(0.27)^{* *}\end{array}$ & $\begin{array}{c}-0.35 \\
(0.17)^{*}\end{array}$ & $\begin{array}{c}-1.05 \\
(0.49)^{*}\end{array}$ & $\begin{array}{c}-0.71 \\
(0.27)^{* *}\end{array}$ & $\begin{array}{c}-0.71 \\
(0.27)^{* *}\end{array}$ \\
\hline Takers of & -51.92 & 103.48 & 82.86 & 38.71 & 103.48 & 103.48 \\
\hline New Generics & $(28.85)+$ & $(28.91)^{* * *}$ & $(16.70)^{* * *}$ & -49.85 & $(28.91)^{* * *}$ & $(28.91)^{* * *}$ \\
\hline $\mathrm{F}$ & 2 & 11 & 12 & 3 & 11 & 11 \\
\hline Sample & all & covered & $\begin{array}{l}\text { covered \& } \\
\text { OOP is copay }\end{array}$ & $\begin{array}{l}\text { covered \& } \\
\text { OOP is coins. }\end{array}$ & covered & covered \\
\hline
\end{tabular}

This table reports the first stages that correspond to the IV analyses in Table 6 †, *, **, and *** represent significance at $10,5,1$, and 0.1 percent. 
Table A5: Effect of Profitability on Benefit Design in Medicare Part D By Plan Enrollment

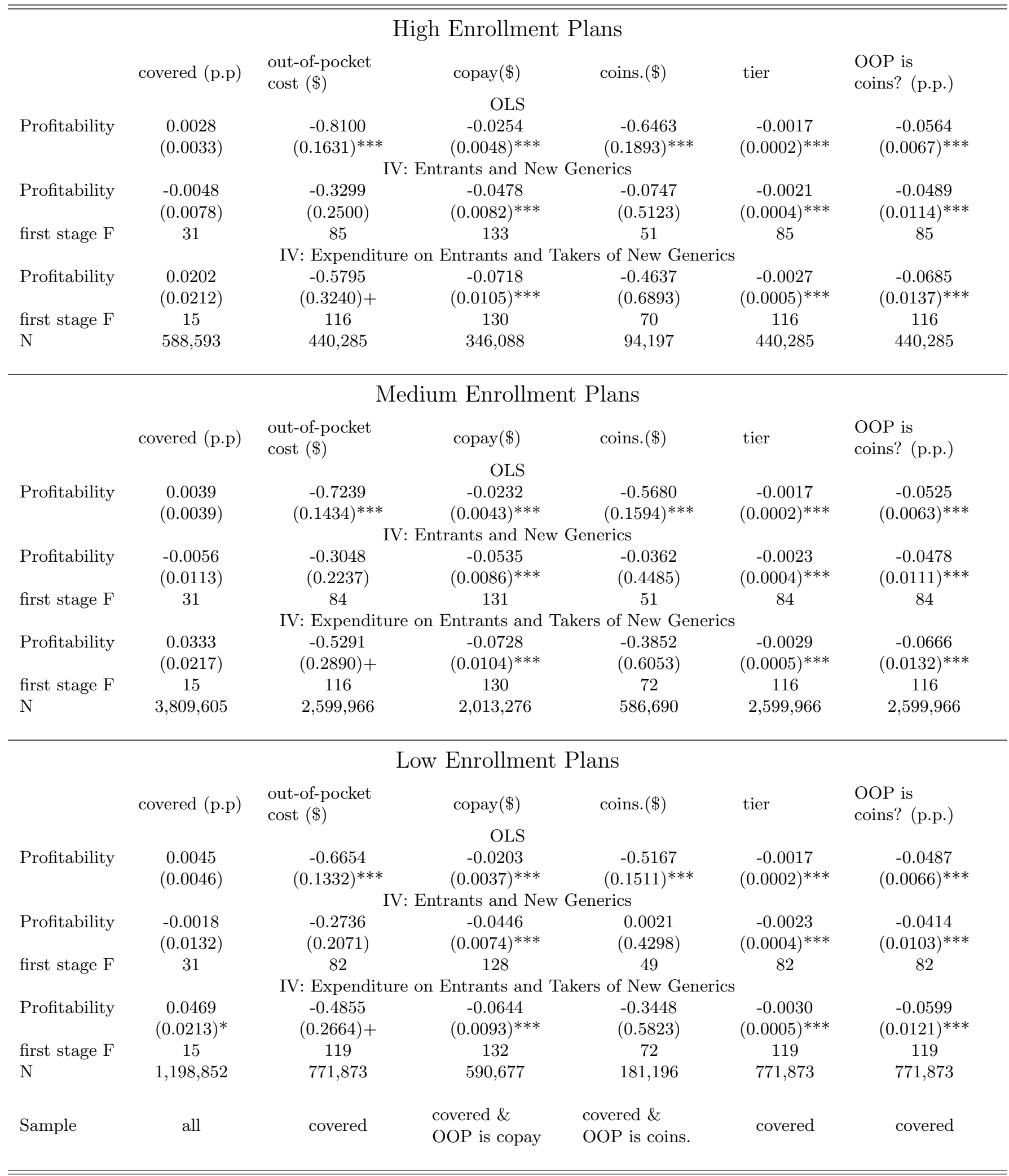

This table reports the results of estimation of Equations 4 across three samples of plans: 163 plans with more than 25,000 enrollees, 1055 plans with between 500 and 25,000 enrollees, and 332 plans with fewer than 500 enrollees. In each panel, the dependent variables are a binary coverage measure or, if covered, the copay or copay as a percentage of list price for each drug in 1550 Part D plans in 2010. The first results are OLS and the remaining are 2SLS with the indicated instruments. Plan dummies are always included. When the outcome is coverage, controls for therapeutic class are included. Standard errors (in parentheses) are clustered on drugs. $\dagger, *, * *$, and $* * *$ represent significance at $10,5,1$, and 0.1 percent. 